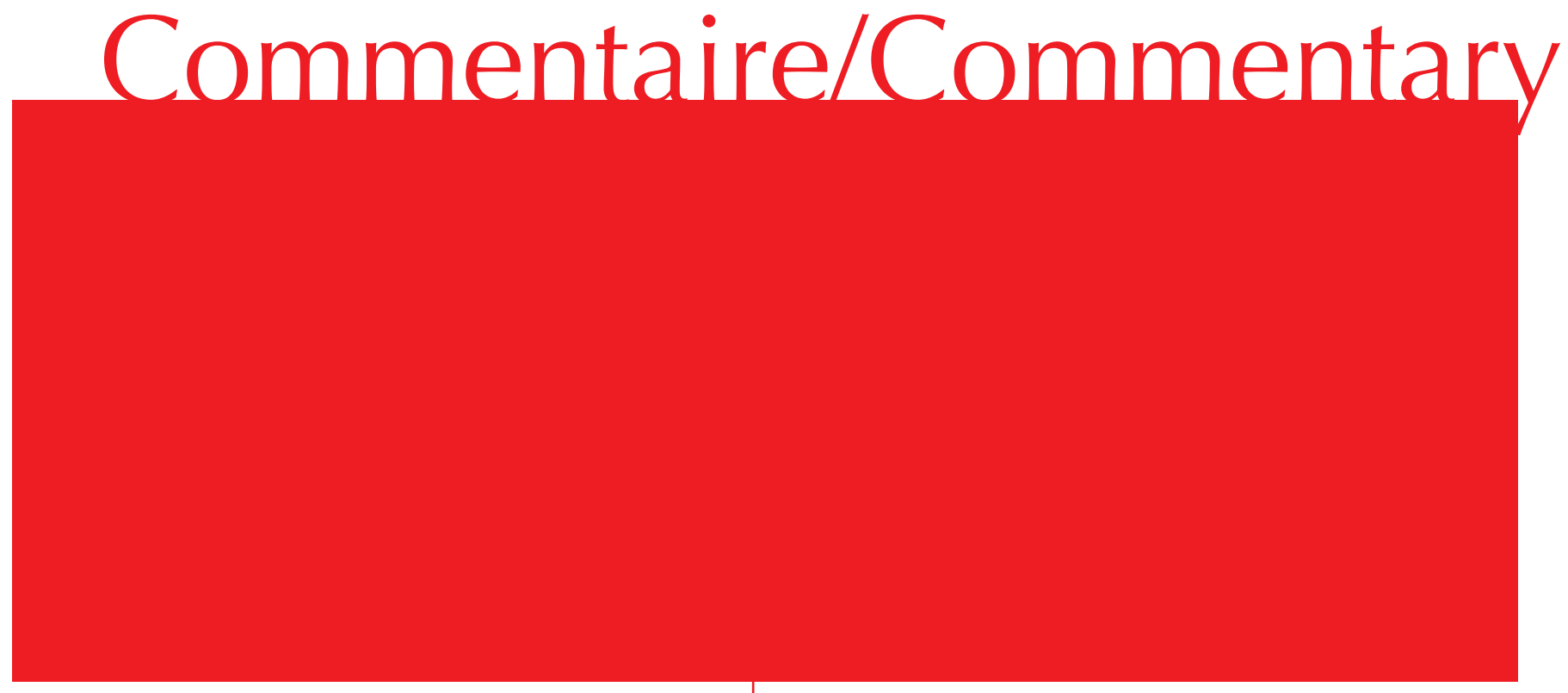

\section{L'Oaristys Noir : le dialogue et l'écoute comme outils dans la prise en charge du deuil des familles des patients atteints de cancer en phase terminale}

\section{CAROL JACQUES}

\section{Introduction}

Par deuil il faut entendre l'ensemble des ritualités qui aident à exprimer la souffrance, la douleur intense et I'affliction que l'on éprouve lors de la perte d'une personne qui nous est chère. Ces rites expriment aussi, pour nous les humains, les évolutions sociétales dans notre relation avec la mort.

Partout dans le monde, les rituels intrinsèques à chaque forme distincte du deuil, ont comme fonction de savoir contenir, guider et circonscrire l'affliction tout en permettant d'ordonnancer également la séparation, autant que la relation, avec la personne disparue.

Vraisemblablement aujourd'hui nous semblons avoir de plus en plus perdu ces symbolismes lesquels peuvent apparaître, pour bon nombre d'entre nous, comme excessifs, voire complètement inappropriés.

Paradoxalement de nos jours, I'expérience de I'homme moderne face à la mort, parait se fragmenter d'une part par la réduction de notre proximité avec le trépas d'autrui et du deuil qui s'en suit et, d'autre part, par un nombre invraisemblable de "séparations » d'objets, de lieux et de personnes que nous expérimentons tout au long de notre existence.

Dans une perspective de société hautement consumériste et dans laquelle un objet perdu peut facilement être substitué par un autre, la mort d'un proche modifie la prise de conscience de ce que représente " une perte », laquelle serait, cette fois-ci, irrémédiable et insubstituable à la fois. 
C'est donc cette disjonction entre le « perdu » et « l'existant » qui contribue aujourd'hui à assécher notre chagrin face à une séparation.

Cela n'enlève pas, bien sur, le fait que la souffrance éprouvée lors la perte d'un être cher constitue interminablement un traumatisme psychique non négligeable. Un heurt qui impacte toujours note expérience intérieure de la souffrance et qui mute dans sa prédisposition à exprimer, plus ou moins explicitement, notre peine psychologique vers l'extérieur, au gré des différentes cultures auxquelles nous appartenons.

$C^{\prime}$ est justement dans ces premiers instants où notre rapport avec le deuil nous confronte avec la difficile épreuve de I'affliction, qu'une prise en charge qualitative fondée principalement sur l'empathie, le dialogue et l'écoute, présenterait un intérêt majeur, autant qu'agissant, pour ceux qui expérimentent cette inexprimable douleur.

En milieu hospitalier la "scène de la vie » ne s'arrête pas au dernier souffle du mourant car, autour de ce dernier, les survivants - sa famille et ses proches - continuent à subsister.

Nous infirmières, nous savons techniquement passer des soins aux patients aux soins mortuaires, cependant nous savons si peu et si mal, gérer la myriade de sentiments que le deuil génère dans la famille du décédé et de son épais corollaire de sentiments si profonds, si méconnus et si divers, qu'il déclenche.

Si humains aussi.

Aujourd'hui, dans nos sociétés développées, la fin de vie d'un patient hospitalisé se trouve loin des rites d'accompagnement qui existaient autrefois. Ces rites lorsqu'ils s'exprimaient dans « l'aménité » d'un foyer, savaient toujours favoriser efficacement l'éclosion et l'imprégnation du deuil.

Ces coutumes étaient jadis déployées afin d'assembler et de particulariser à la fois, dans un emplacement intime et dépourvu d'aspérités, chaque membre d'un même foyer, ou d'une même collectivité réunis, durant l'encours du deuil, dans une équivalente expérience de la douleur.

Or, qu'elle survienne au sein d'une habitation ou dans un hôpital, la mort d'un individu ne sera pas vécue avec la même accentuation par sa famille.

Dans nos services de soins si hautement techniques, la mort est considérée souvent comme un revers de la médecine. Ce dérisoire refus de l'échec provoque parfois tant de conséquences dommageables non seulement pour les familles des disparus, mais également sur nous, soignants hospitaliers.
Il nous arrive aussi de ressentir de la culpabilité, du chagrin, de l'impuissance, de la peine, de la détermination, des regrets, de la fatalité, de la souffrance et de la peur ...

Une appréhension que parfois nous ressentons lorsqu'il faut, par exemple, à coté du médecin, participer à l'annonce de la mort $d^{\prime}$ un patient à ses proches.

De notre propre angoisse de soignants lorsque nous percevons la nécessité d'exprimer, avec nos propres formules chancelantes, les condoléances à la famille d'un patient.

De cette manière hésitante aussi que nous avons " de dire » un décès, pour être explicitement entendus par tous ceux, qu'autour de nous, le désirent.

Comment faire? Comment s'y prendre ? Sommes-nous dans nos rôles lorsque nous exprimons cela? Avec quels « dits »?

A I'hôpital pourtant, cet accès très intime car suivi, aux proches du mourant incombe aux soignants que nous sommes car nous avons pu discerner, mieux que tout autre professionnel de la santé, les évolutions de l'affection du patient.

Cette précise cognition des contextes cliniques que nous soignants avons de la maladie, sait instinctivement nous guider, lorsque cela est le cas, pour préparer progressivement les familles à l'avènement du "sans retour possible » du proche et qui préfigure, à terme, leur endeuillement à venir.

Mais pour que l'aide et la prise en charge puissent se révéler efficaces, encore faut-il que nous puissions connaître les mécanismes de la tristesse, les signifiants du deuil et de ses dangereuses conséquences induites lorsque ces processus ne peuvent, de manière positive, être ni élaborés, ni expérimentés par la famille.

Bon nombre d'études anthropologiques[1] ont mis en évidence les différentes modalités par lesquelles chaque société a su développer un système de croyances sachant offrir une explication de la mort en même temps qu'elles proposent des rites qui apportent un support social pour mieux exprimer, en les rassemblant, les émotions qui jaillissent à la suite d'un décès.

Tout au long de la période de la complainte nos us sociaux déterminent les rôles que chaque membre de la famille du disparu doit revêtir. Ces usages fixent également la nature et la durée des restrictions aux activités que les personnes les plus liées au disparu, se doivent d'appliquer.

Or aujourd'hui ces rituels sont fréquemment vite bâclés et vidés de leur ancestral sens cathartique. 
Sans ces repères culturels et le soutènement du " collectif » dont jadis les "endeuillés » bénéficiaient, les proches du défunt paraissent de plus en plus fragilisés avec la menace de glisser indiciblement dans la solitude. Un confinement psychique où l'angoisse semble tout juguler.

Les rites funéraires[2] liés au deuil, aident donc à mieux séparer les morts des vivants et concourent à apaiser les troubles de l'inquiétude que nous, vivants, éprouvons face à la mort.

Ces rituels facilitent aussi le détachement tout en permettant la création de " canaux " par lesquels continue à s'exercer une « communication» des proches avec leur disparu.

Les rituels aident également à réduire l'état de confusion et de la douleur psychologique (affliction) dans lesquels résident ceux qui expérimentent le deuil.

Le chagrin, la peine, la tristesse sont des réactions naturelles et physiologiques. Elles indiquent un ensemble d'étapes liées à la souffrance psychique et qui ont habituellement tendance à s'affaiblir et à décroître avec le temps.

Mais parfois ces apaisements semblent impossibles à s'établir. L'affliction alors se transforme en profond traumatisme permanent lequel sait encourager, dans bon nombre de cas, I'apparition d'une pathologie psychiatrique.[3]

Pour mieux comprendre les étapes psychologiques du deuil, il est possible de les schématiser en deux " espaces " principaux, sans pour autant omettre que ces différents cycles peuvent s'assembler voire s'amalgamer, de manière aléatoire et non prévisible.

\section{Avant l'avènement du deuil :}

- Choc;

- Négation ;

- Affliction anticipative ;

- Isolement ;

- Anxiété ;

- $\quad$ Peur du futur.

\section{Au moment où la mort survient :}

\section{- Choc;}

- $\quad$ Angoisse - Recherche ;

- $\quad$ Colère - Sens de culpabilité ;

- Désespérance - Sens d'abattement.

Immédiatement après le décès les proches du défunt transitent par une étape d'étourdissement total où l'on entre comme dans une espèce de dimension irréelle et dans laquelle le sujet semble incapable de penser et de réfléchir.

Il se sent alors vidé, affaibli, éprouvé jusqu'à occasionner des réactions somatiques comme par exemple, dans certains rares cas - des évanouissements, des palpitations, etc.

Après de quoi se manifeste l'étape où, la personne endeuillée, laisse libre cours à son angoisse, aux regrets, au chagrin, aux doutes.

Elle recherche et elle pense interminablement à la personne disparue ...

La recherche de la personne décédée est alors fréquemment accompagnée de manière plus ou moins intense par un temps de transition où l'on éprouve, parfois, de la colère envers chaque chose, envers tout chacun.

II n'est pas rare que, dans certains cas, cette colère puisse alors être tournée envers soi-même lorsque par exemple l'on se sent, d'une manière ou d'une autre, à tort ou à raison, responsable de la disparition d'un proche et/ou que l'on éprouve, de ce fait, de la culpabilité.

Ainsi, ce refus de la mort s'exprime en tant qu'expression de l'impuissance ressentie face aux brutales matérialités de la vie.

La recherche du disparu, par ses survivants, se matérialise ainsi dans la négation de la réalité.

Intervient alors un état d'apathie dans lequel le sujet glisse dans une forme marquée d'asthénie.

Cette asthénie[4] qui s'exprime chez la plupart des sujets empêchés de vivre rituellement un deuil, incite subséquemment la survenue du «déplaisir » à la vie.

Si à ce moment paroxystique dans lequel s'exprime l'intense douleur qu'éprouvent ceux qui font l'expérience de la mort d'un proche, n'interviennent pas des prises en charge efficaces par l'écoute et par le dialogue, les risques sont alors de voir s'accroitre le taux élevé de mortalité chez des sujets les plus sensibles, voire fragiles ou, plus ordinairement, un risque étendu de survenue d'une maladie psychiatrique telle que la dépression.

Les causes qui pèsent dans le dépassement, ou pas, de cet écueil critique, peuvent-être résumées ici en trois groupes distincts :

- Les facteurs liés aux origines et aux modalités du décès comme par exemple la durée de la maladie mortelle ou de l'agonie du patient, I'âge de la personne décédée, le caractère de cette agonie (avec ou sans douleur, délires, etc.), l'aspect de la dépouille mortelle ;

- Les facteurs préexistants à la maladie du disparu comme, par exemple, le type de relation du survivant avec le décédé, son degré de parenté, son 
degré de dépendance psychologique vis-à-vis de la personne décédée, le degré d'autonomie financière, économique et sociale du décédé, les différents sens de culpabilité accumulés, le sexe du survivant, sa classe sociale, la présence, ou pas, d'autres expérience du deuil, la préexistence, ou pas, de facteurs culturels (ou cultuels) spécifiques permettant favorablement l'expression pleine et entière du deuil ;

- Les facteurs qui se seraient déterminés tout au long d'une longue maladie mortelle et de la phase terminale de celle-ci, comme par exemple le type de communication entre le mourant et la famille, le degré de conscience des proches du disparu quant à l'irréversibilité de sa maladie et de l'imminence de la mort, la qualité, ou pas, de la communication entre la famille et l'équipe soignante (médecins, infirmières ...), le caractère de la prise en charge prodiguée par I'hôpital au même parent du mourant.

A cela, différentes études ont permis d'y opposer au moins trois autres théories principales psychologiques capables celles-ci, de favoriser avantageusement le dépassent du deuil:

- La théorie biologique selon laquelle la mort (comme toute autre perte) parvient à sectionner un lien d'attachement et replace, de ce fait, la question de la survie du sujet survivant.[5] Dans cette optique, le deuil alors ne serait rien d'autre que le processus tendant à résoudre le problème d'une survie qui ne serait plus garantie.

Selon cette théorie, la personne en deuil, serait prédisposée à mettre en œuvre un complexe comportement de recherche de la personne disparue, comme s'il s'agissait de vérifier que la mort se soit incontestablement réalisée.

Une façon aussi pour le sujet, de valider l'impossibilité de récupérer cette perte.

Frustré dans sa propre survie, le proche du défunt accomplirait une "prise de distance » envers celui qui n'est plus disponible pour les fins de sa propre survivance.

Une situation qui changerait ainsi l'identité de " l'indemne » en le rendant nouvellement disponible à l'établissement de "liens » nouveaux aptes à sa survie.

- La théorie psychanalytique selon laquelle la recherche spasmodique de la personne disparue aurait, dans sa mise en œuvre, un caractère défensif car réfutant la perte subie.

Cette recherche[6] du deuil par le refus de la disparition de l'être cher, ne comporterait pas une volonté de « prospection » de celui qui n'est plus, mais témoignerait plutôt d'une acceptation du caractère inexécutable de toute tentative de le retrouver.

Le relâchement de ce lien avec la personne perdue ne serait possible que grâce à une " réparation " de la perte subie. Une réparation qui consisterait à faire revivre, cette fois de l'intérieur, " l'objet/sujet» perdu afin de stimuler sa progression vers une réalité extérieure qui se traduirait, pour le survivant, par une forme d'identification avec son disparu afin d'en faciliter ensuite, I'introjection.

- La théorie existentielle, enfin, et selon laquelle le but du deuil serait préférablement de nous faire oublier nos morts au lieu que de nous adapter à des nouveaux liens possibles avec eux. Selon cette théorie[7] cette forme de I'oubli n'entrerait pas en crise avec la mort du proche mais avec le sens même de la vie car, avec la mort, la « nature » parviendrait à mettre en crise " I'histoire».

Le deuil est ici décrit comme une atteinte de la « présence ", c'est-à-dire une crise propre à l'existence humaine laquelle est pensée maintenant non plus comme une vie seulement biologique ou encore subjective mais, au contraire, comme une vie pourvue d'un sens que nous-mêmes aurions érigé et qui poursuivrait sa propre construction culturelle intrinsèquement à notre propre destin.

Dans l'une comme dans l'autre, dans ces trois pensées divergentes du deuil il s'agirait, de parvenir à récupérer, par la mort d'un proche, le sens que nous aurions perdu de I'existence.

Une disposition intérieure capable de mieux nous aider à cheminer dans l'avènement de la mort tout en donnant, par opposition, un (nouveau) sens à la vie.

En définitive, dans le deuil, il s'agit non pas d'essayer de faire revivre en nous nos propres morts, mais plutôt de les faire culturellement ... mourir dans nos propres existences.

Vivre précisément le deuil aujourd'hui désignerait d'être enfin capables de pouvoir oublier les proches disparus afin que ceux-ci, n'étant plus définitivement présents, puissent être mieux ... remémorés.

Une manière aussi d'œuvrer enfin, pour que nos morts puissent à jamais être situés et avec précision ... dans le passé.

Encore faut-il être capable de manœuvrer dans ce sens.

Encore faut-il que nous, soignants, puissions aider autrui à accomplir ce(s) deuil(s) nécessaire(s) en identifiant avec précision son approche exacte du deuil ainsi que ses propres nécessités.

Pour nous, infirmières, assister une personne expérimentant I'affliction constitue un défi immense, car l'approche d'une personne en deuil implique de connaître l'ensemble des facteurs qui interagissent sur l'évolution des souffrances, qu'elles soient de nature biologiques, psychologiques ou encore sociales.

Savoir également le type d'accompagnement attendu par la 
famille endeuillée et qui serait composé d'un subtil dosage : d'empathie, d'écoute et de dialogue.

Connaître aussi les modalités phycologiques qui peuvent constituer les forces, ou les freins, avec lesquels la personne en deuil s'apprête à affronter cette douloureuse période.

Ils n'existent malheureusement ni des " recettes » ni des comportements standardisés pour une approche cathartique favorable du deuil, mais cette perception de l'affliction humaine faisant suite au décès d'un être cher, doit nous autoriser, nous soignants, à l'élaboration d'une attitude utile autant que spécialisée pour aider la famille du disparu à affronter cette problématique lourde de significations autant que de conséquences.

D'être capables en somme, nous soignants, de posséder des pratiques adéquates et attendues de la société à laquelle nous appartenons. De maîtriser aujourd'hui l'emploi d'instruments primordiaux afin de réduire cette perception de déshumanisation, de froideur et d'éloignement des êtres entre eux.

Etre davantage des " dispositifs aidants » non seulement visà-vis du mourant, mais également pour ses intimes.

Je pense notamment aux cas que quotidiennement je côtoie dans mon activité d'I.D.E au sein du plus grand centre en Europe de lutte contre le cancer : "Gustave-Roussy » et au sein duquel nombreuses sont les situations où des patients endurent une pathologie cancéreuse en phase terminale.

Dans ces lieux d'exercice du soin à haute portée technique médicale et où certains patients ne sont plus dans une expectative de guérison mais seulement dans l'attente de recevoir à la fois du confort et du respect, la famille, heureusement, n'est jamais abandonnée à elle-même.

Prendre en charge une famille endeuillée comporte, pour le soignant, de cheminer vers d'attitudes thérapeutiques qui requièrent de savoir modifier ses approches et ses motivations en matière de soin.

Des pratiques trop encore amarrées à la séculaire notion du : " soigner pour guérir " plutôt qu'à celle, plus inusitée, de: « soigner pour ... prendre soin ».

Nous les infirmières nous représentons la figure sanitaire dans laquelle se concrétisent le mieux ces caractéristiques capables de fournir soutien et réconfort non seulement aux malades mais également à leur entourage.

Pour agir ainsi nous adossons nos pratiques aux énoncés relatifs du Décret n² 2004-802 du 29 juillet 2004[8] lequel fixe le périmètre de nos agissements et de nos compétences de soignants.

Nous pouvons percevoir dans ce texte les missions et les compétences qui sont les nôtres et qui reconnaissent, dans notre profession, les capacités et les connaissances nécessaires $d^{\prime}$ interagir efficacement et de manière complémentaire aux médecins - comme aux autres opérateurs de la santé - et non plus de manière accessoire à ceux-ci.

Car dans l'épreuve de la maladie comme aussi dans celle de la souffrance expérimentée par les proches du disparu, nous paraissons les mieux-à-même pour concéder une proximité gorgée d'empathie, d'écoute et de dialogue.

Cette prédisposition aux complexités du " soin » et de la prise en charge de l'humain, nous permet de développer des relations subtiles fondées principalement sur la confiance car nous, infirmières et infirmiers, nous sommes, bien plus que tout autre opérateur sanitaire, davantage plus impliqués, davantage plus inclus, davantage plus engagés aussi.

Nous pouvons prévenir les refoulements, les inhibitions autant que les négations des sentiments nocifs qui peuvent jaillir parfois, lors des phases aigues des réactions au deuil.

Mais sur ces formes toutefois si distinctives du « savoir soigner ", nous paraissons également ici, dans les territoires Hexagonaux où s'exercent nos compétences, avoir pris un certain retard.

Depuis plusieurs années en effet, dans certains hôpitaux anglo-saxons qui traitent des pathologies lourdes comme le cancer, est émergé une figure nouvelle : celle de l'infirmière "Counselor »,[9] dont l'une de ses fonctions principales consiste à atténuer les angoisses et les incertitudes, voire les défiances, qui pourraient inopportunément s'insinuer entre les familles des patients et les soignants... Plus généralement elle intervient là encore où les diagnostiques vitaux semblent être engagés.

Pas vain donc que de militer pour que chez nous aussi, des telles compétences à l'écoute et au dialogue, à défaut de l'existence de spécialisations idoines, puissent faire l'objet de modules de formations supérieures à la fois durables autant qu'accessibles.

Etre en permanence en contact direct avec la mort, comme nous le sommes, comporte de savoir à la fois affronter et gérer un double aspect de cette situation : d'une part de savoir apporter qualitativement les soins thérapeutiques exigés et, d'autre part, s'interroger au sujet de la question qui nous rapproche quotidiennement, en tant qu'individus, à l'expérience de la mort et de la perte. 
Pour assister le patient à affronter l'idée de la mort et soutenir ses proches dans le mûrissement de l'idée de son absence, il est important d'avoir parfaitement intériorisé en nous-mêmes la perspective de notre propre mort comme faisant partie intégrante de la vie.

Acquiescer à cela ne signifie pas réduire voire annuler toute autre expérience de la vie, mais au contraire cela comporte d'être mieux stimulés dans les engagements, les choix et les expériences hétérogènes que la vie nous propose.

Il me semble ainsi nécessaire d'œuvrer au développement $d^{\prime}$ une préparation personnelle et professionnelle fondée sur la recherche culturelle et sociologique du deuil autant que sur les uniques atouts à la gestion psychologique vis-à-vis de la mort et du mourir.

Une exigence d'un savoir-faire et d'un savoir-être plus élargis, et qui mieux viseraient la reconnaissance d'une réévaluation de nos propres codes et de nos propres aperçus que nous tenons de nos connaissances techniques lesquelles doivent s'encrer plus vigoureusement encore sur nos inséparables valeurs éthiques de soignants.

Un appétit de progrès davantage structurant pour notre métier et où, dans les trois moments clés où tout se joue : avant, pendant et après le décès du patient, les familles et le soignant, ne sauraient plus être disjoints, mais conjugués.

Notre rôle de soignants dans la gestion du deuil nécessiterait, en conséquence, de mieux considérer les pourtours complexes attenants à l'éducation, à l'information, le soutien émotionnel, la gestion de la crise aigue de la douleur liée au deuil, l'identification des familiaux à " haut risque », et à la qualité de assistance apportée à la famille du disparu, à bref et à long terme.

Pour ce faire, pour mieux le faire, nous soignants, exerçant en milieu oncologique devrions ainsi être présents dès le premier instant où le médecin communique à la famille I'irrévocabilité d'un diagnostic négatif et aussi lorsqu'il expose, le déroulé des soins qui en découleront.

Il est fondamental qu'à cette étape précise, où le mot irréversibilité est prononcé, que l'infirmière sache apprécier exactement la situation sanitaire du patient afin de l'éclairer si besoin en est, sans pour autant faire l'impasse sur son rôle indispensable de médiation auprès de la famille afin d'apporter à celle-ci, des réponses claires, indispensables et complémentaires à une meilleure capacité, pour les proches du patients, à désamorcer toute incompréhension ultérieure possible.
En permettant ainsi à la famille d'exprimer librement leurs profondes interrogations ou encore en les écoutant dans leurs souffrances, le soignant se trouverait ainsi dans une position unique pour faciliter la mise en œuvre du processus du deuil car, si ce " temps " de résilience sait se manifester précocement - phase du deuil anticipatoire - cela peut permettre à la famille une meilleure expression de ses propres perceptions des émotions profondes et si fréquemment tues, avant que la disparition du proche ait lieu.

Encore faut-il que nous sachions résister aux indicibles formes de douleur et de leur lourd cortège de tristesses, de peines et de larmes que le deuil, en ces pénibles circonstances, occasionnen et qui tant peuvent, nous soignants aussi, nous « altérer ».

\section{Conclusion}

Nous remarquons à quel point il est primordial, pour les soignants que nous sommes, qu'une communication active puisse être durablement maintenue avec le patient comme avec sa famille afin d'entretenir continuellement le sentiment d'espérance tout au long du deuil.

Exhorter aussi que le deuil puisse être conçu et mis en œuvre à tout moment par la famille de manière ante ou post séparation irrévocable du malade.

Ensuite pour que cette notion "d'espérance » puisse être fondée sur la confiance, et qu'elle sache également se propager, par capillarité, à l'ensemble des membres de la famille comme aux autres proches du disparu, lesquels peuvent être, à leur tour, un non négligeable " support » pour nous soignants car pour nous aussi «perdre » un patient, n'est jamais une chose anodine.

Les proches qui assistent et les soignants qui maîtrisent le soin prodigué au mourant ne doivent plus être scindés face aux difficultés et aux conséquences de l'engagement que le deuil comporte.

Enfin, en France, comme c'est de plus en plus le cas en Europe, nos institutions de formation aux soins infirmiers doivent encore plus corroborer, intensifier et diversifier nos capacités et nos sensibilités à la prise en charge de la famille.

C'est elle qui expérimente à chaque étape de la vie l'indicible périple ontologique du deuil. Nous devons encore plus nous impliquer intensément dans cette voie, comme nous le faisons pour les autres soins. Mieux assimiler d'abord, pour produire ensuite dans nos pratiques cette étrange approche de la vie par la mort, participe à faire de nous des acteurs thérapeutiques capables d'atténuer les heurts sociétaux 
induits par un monde que nous trouvons de plus en plus déshumanisé, plus insensible, plus froid et plus distant.

Par nos compétences techniques nous savons œuvrer chaque jour afin que la mort puisse advenir dans la dignité, avec des souffrances physiques et psychologiques limitées. Et c'est bien.

A nous aussi toutefois de ne jamais omettre, dans nos «prises en charges » de tous les instants, d'influer de manière douce et plus humaine afin de mieux favoriser l'accomplissement de ce cycle si complexe, si douloureux et pourtant si nécessaire, relatif à la transition par le deuil pour tous ceux qui survivrons au décès d'un proche.

\section{Références}

1.Faivre D. (éd.). La Mort en question. Approches anthropologiques de la mort et du mourir, Ed. Erès poche ; (Espace éthique). 2013.

2.Satet P. (dir.) Le grand livre de la mort à I'usage des vivants. Paris, A. Michel. 2007.

3.Freud S. Deuil et mélancolie. Paris. Payot. 2011. (1917).

4.Baudry P, Juedy H.-P. Le Deuil impossible : fenêtre sur la mort. Paris. Eshel, 2006.

5.Debout M, Cettour D. Sciences et mythologie du mort. Paris, Vuibert. 2006.

6.Bacque MF. L'un sans I'autre. Psychologie du deuil et de la séparation. Paris. Larousse. 2007.

7.Jolivet R. Le problème de la mort chez M. Heidegger et J.-P. Sartre. Abbaye Saint-Wandrille. Ed. de Fontenelle. 1950.

8.http://www.legifrance.gouv.fr/affichTexte.do?cidTexte=JOR FTEXT000000787339 consulté le 10.9.2014

9.Formarier M, Jovic L. Les Concepts en Sciences Infirmières. Ed. Mallet Conseil. (2ème éd.). ARSI. Lyon. 2012

Pour contacter l'auteure:

Carol Jacques

Infirmière Bachelière

Services Hématologie-Endocrinologie

Gustave Roussy - Cancer Campus (Grand Paris)

95805 - Villejuif - France

Courriel : carol.jacques88@gmail.com 